\title{
Transposable elements and genomics
}

\author{
Nam-Soo Kim
}

Received: 16 December 2014/ Accepted: 18 December 2014/Published online: 9 January 2015

(C) The Genetics Society of Korea and Springer-Science and Media 2015

Once described as selfish DNA, transposable elements (TEs) have been revisited due to a plethora of new genomic information. TEs are considered indispensable genomic treasures during eukaryotic species evolution and supply raw materials for evolution by creating novel variation and allelic diversity (Volff 2006; Oliver et al. 2013). TEs are found ubiquitously with high copies in nearly all eukaryotic species. TEs are often present in major genomic denizens in some species by occupying more than half of the genome, contributing to the $\mathrm{C}$-value paradox in eukaryotes. In this issue, six articles are published describing several aspects of transposable elements.

Four review articles and two research articles are presented in this special issue. TEs are classified into class I TEs and class II TEs based on transposition mechanisms. Class I TEs are retrotransposons that retrotranspose via RNA intermediate semi-conservative manner. Class II TEs transpose via DNA intermediate conservative manner. Rajput (2015) described class I retrotransposons as the intrinsic genomic evolutionist as "Retrotransposons: the intrinsic genomic evolutionist". He reviewed essential information on retrotransposons. Retrotransposons are major agents in genome evolution and diversity by inducing rearrangements and restructuring genomes. Retrotransposons affect many aspects of biological organization, from gene regulation to genome obesity, which are critical in shaping current genomes. He also discusses the similarity between retrotransposons and endo-retroviruses, bringing the retrotransposons into a closer relationship to

\section{N.-S. Kim $(\bowtie)$}

Department of Molecular Bioscience, Institute of Bioscience and Biotechnology, Kangwon National University, Chuncheon 200-701, Korea

e-mail: kimnamsu@kangwon.ac.kr; nsk6472@yahoo.ca understand phylogenetics. Bae et al. contributed "Biological changes of transposable elements by radiation" (Bae et al. 2015). Human endogenous retroviruses (HERV) are a group of LTR (long terminal repeat) retrotransposons in the human genome. Transcriptionally-active TEs can move around in the genome and cause genome instability. HERV insertion into critical genes may lead to the progression of some diseases, including cancer. These authors reviewed cases of human disease due to HERV insertion. Radiation therapy manifests diverse, complex, and multicellular responses, including unmasking epigenetic suppression that switches silenced TEs into active ones. In the review, the authors summarized the recent understanding on radiationinduced DNA double-strand breaks (DSBs) and subsequent non-homologous end joining (NHEJ) repair. They also presented their recent results on activation of $\mathrm{L} 1$ elements by radiation in cancer cells, in which the methylation level of L1 was increased by relatively low radiation doses. Based on these results, the authors proposed that radiation exposure may lead directly to mutation as well as to methylation changes in TEs, which are considered important factors in cancer radiation therapy. Lee et al. contributed "Composition and evolutionary importance of transposable elements in humans and primates" (Lee et al. 2015). Primates, including humans, are not immune to the evolutionary impact of TEs on their genomes. Approximately half of the sequences of primate genomes are TErelated sequences. In their review, comparisons of $\mathrm{TE}$ constitution among primate species are extensively explained, and TE dispersions in the genome were analyzed between human and primates. These authors proposed that TE-driven structural variations are a major force in primate evolution. Roy et al. examined TEs in different aspects of molecular markers in "Marker utility of transposable elements for plant genetics, breeding, and 
ecology" (Roy et al. 2015). Genetic variation is primary in genetics, breeding, and ecology. With the development of polymerase chain reaction (PCR) in molecular biology, various molecular marker systems have been developed in last two decades. Conventional marker systems detect mutations derived from base substitutions, insertions/ deletions (in/dels), or structural changes in chromosomes. TE-based molecular marker systems detect variations derived from TE transposition. Transposon display (TD) is a modified technique of conventional amplified fragment polymorphic DNAs, but the molecular variations detected by TD were more variable than AFLP variations. Unidirectional transposition of retrotransposons rendered retrotransposon-based markers highly informative in tracing the genetic history of species divergence in phylogenetic analyses.

Kwak et al. presented a novel research article entitled "Evolutionary fate of SVA2 elements in primate genomes" (Kwak et al. 2015). SVA refers to SINE/VNTR/Alu elements. In the human genome, approximately 2,800 SVA elements have been identified across all chromosomes. SVA elements are considered some of the youngest retrotransposons in primates. It was estimated that SVA elements integrated into the primate genome approximately 25 million years ago after divergence of Old World monkeys and other ape lineages. In the Kwak et al. (2015) study, SVA2 element shared sequences with VNTR, but there was no obvious sequence sharing of SINE-R with other SVA elements. These researchers illustrated SVA2 evolution and the impact of the SVA2 element on human, chimpanzee, gorilla, and rhesus monkey genomes. They found no species-specific SVA2 elements, indicating that there are no retrotranspositionally-active SVA2 elements in primate genomes. Moreover, they identified two SVA2 elements in humans that have miRNA target sites, suggesting they might be involved in regulation of gene expression and gene-related human diseases. YJ Kim presented her recent findings on transposable element-derived
microRNAs in the article "Evolutional and functional analysis of four microRNAs (miRNAs) derived from transposable elements" (Kim 2015). Previous studies showed that miRNAs originated from TEs, and in return, TEs are important for expression and regulatory network formation of miRNAs. In Kim's article, the expression patterns of four TE-derived miRNAs, miR-330, miR-648, miR-1254, and miR-1825, were conserved in various species. Moreover, these TE-derived miRNAs showed inhibitory effects on the expression of their own target genes: the tumor suppressor MLL2 and the oncogene ARHGEF12. These results confirm that TEs generate miRNA sequences, which are important in regulating gene expression.

\section{References}

Bae JI, Kim HS, Kim TO, Yi JM (2015) Biological changes of transposable elements by radiation: recent progress. Genes Genomics 37. doi: 10.1007/s13258-014-0256-Z

Kim YJ (2015) Evolutional and functional analysis of four microRNAs derived from transposable elements. Genes Genomics 37. doi: $10.1007 / \mathrm{s} 13258-014-0255-0$

Kwak Y, Kim YJ, Xing J, Han K (2015) Evolutionary fate of SAV2 elements in primate genomes. Genes Genomics 37. doi: 10.1007/ s13258-014-0241-6

Lee HE, Eo JE, Kim HS (2015) Composition and evolutionary importance of transposable elements in human and primates. Genes Genomics 37. doi: 10.1007/s13258-014-0249-y

Oliver KR, McComb JA, Green WK (2013) Transposable elements: powerful contributors to angiosperm evolution and diversity. Genome Biol Evol 5:1886-1891

Rajput MJ (2015) Retrotransposons: the intrinsic genomic evolutionist. Gene Genomics 37. doi: 10.1007/s13258-014-0250-5

Roy NS, Choi JY, Lee SI, Kim NS (2015) Marker utility of transposable elements for plant genetics, breeding, and ecology: a review. Genes Genomics 37. doi: 10.1007/s13258-014-0252-3

Volff JN (2006) Turning junk into gold: domestication of transposable elements and the creation of new genes in eukaryotes. Bioessays 28:913-922 